\title{
Los grandes ensayos clínicos de anticoagulación en fibrilación auricular pueden trasladarse a la práctica diaria
}

\section{Objetivo}

Evaluar el efecto del uso de warfarina en fibrilación auricular (FA) y el riesgo de tromboembolismo, hemorragia y muerte en pacientes bajo el cuidado habitual.

\section{Diseño}

Estudio prospectivo de cohorte.

\section{Ámbito}

Sistema de Salud Integrado de California del Norte.

\section{Población}

Fueron elegibles todos los pacientes mayores de 18 años con diagnóstico de FA entre el 1 de julio de 1996 y el 31 de diciembre de 1997 (visita índice). Se excluyeron pacientes con FA valvular, reversible o transitoria. De los pacientes con FA no valvular, se incluyeron aquellos sin contraindicación para anticoagulación.

\section{Intervención}

Los pacientes fueron seguidos en forma prospectiva, desde la visita índice hasta el 31 de Agosto de 1999.

Se determinaron: factores de riesgo para ACV, uso de warfarina y porcentaje del tiempo en rango de anticoagulación. Durante el seguimiento se registraron eventos tromboembólicos y mortalidad. Se utilizaron bases de datos del ambulatorio e internación, farmacia y laboratorio. Además se revisaron historias clínicas y registros estatales de mortalidad.

\section{Medición de Resultados Principales}

Accidente cerebrovascular (ACV), embolia periférica, hemorragia y muerte relacionada con el uso de warfarina y comorbilidades.

\section{Resultados}

Se incluyeron 11.526 pacientes: $43 \%$ eran mujeres y la edad media era de 71 años (23\% >80 años). El $80 \%$ de los pacientes en tratamiento con warfarina era seguido por programas de anticoagulación. Durante el seguimiento estuvieron en rango (RIN 2-3) el $63 \%$ del tiempo. Los resultados se resumen en la tabla 1.
Tabla 1. Tasa de eventos y anticoagulación

\begin{tabular}{l|c|c|c}
\multicolumn{1}{c|}{ Evento } & $\begin{array}{c}\text { Tasa de eventos con } \\
\text { warfarina*(IC 95\%) }\end{array}$ & $\begin{array}{c}\text { Tasa de eventos sin } \\
\text { warfarina*(IC 95\%) }\end{array}$ & $\begin{array}{c}\text { RRR/ARR } \\
\text { (IC 95\%) }\end{array}$ \\
\hline ACV + embolias perifericas & $1,11(0,94-1,31)$ & $1,88(1.65-2,14)$ & $51 \%(39-60)^{* *}$ \\
\hline Mortalidad & 4.46 & 5.33 & $16 \% \%^{* \star}$ \\
\hline Hemorragia intracraneal & $0,46(0,35-0,59)$ & $0,23(0,16-0,34)$ & $49 \%(31-78)^{* * *}$ \\
\hline
\end{tabular}

* Eventos por 100 personas/año ** RRR $=$ reducción de riesgo relativo ${ }^{* * *}$ aumento de riesgo relativo

El tratamiento con warfarina se asoció a una reducción del riesgo de tromboembolismo del $51 \%$ (IC95\% 39-60) tanto en presencia como en ausencia de factores de riesgo para ACV. Un análisis caso-control anidado estimó que este efecto es aún mayor (64\%) cuando se compara con no recibir tratamiento antitrombótico. Dos tercios de los pacientes que tuvieron un ACV y recibían warfarina tenían un RIN <2. La anticoagulación se asoció también a una disminución del riesgo de muerte por todas las causas (riesgo relativo ajustado 0.69 , IC95\% $0.61-0.77$ ) La hemorragia intracraneal fue poco frecuente, pero la tasa fue moderadamente más alta en aquellos pacientes que tomaban warfarina en relación a los que no la tomaban (0.46 vs. 0.23 por 100 personas/años, respectivamente). No hubo asociación con un incremento en el riesgo de hemorragia mayor no intracraneal. El efecto fue similar cuando los pacientes con contraindicaciones al inicio fueron analizados por separado o conjuntamente con aquellos sin contraindicaciones.

\section{Conclusión}

La warfarina es muy efectiva en la práctica clínica para prevenir el ACV isquémico en pacientes con fibrilación auricular mientras que el incremento absoluto en el riesgo de hemorragia intracraneal es pequeño. Los resultados de los estudios aleatorizados de anticoagulación pueden trasladarse correctamente al cuidado de los pacientes con FA.

Fuente de Financiamiento: Beca de investigación de servicios de salud pública del National Institute of Aging y beca Eliot B. y Edith C. Shoolman del Massachusetts General Hospital.

\section{Comentario}

Los resultados de este estudio son similares a los reportados en la literatura. Estudios realizados tanto en prevención primaria como secundaria demuestran que la warfarina reduce en $2 / 3$ el riesgo de ACV mientras que la aspirina ${ }^{1}$ es mucho menos eficaz ${ }^{2}$ Resultados de estudios recientes como el $\mathrm{AFFIRM}^{3}$ y el $\mathrm{RACE}^{4}$ hablan ya de la importancia de la anticoagulación a largo plazo en los pacientes con FA inclusive luego de restablecido el ritmo sinusal.

Sin embargo existen dudas acerca de si los resultados de los grandes ensayos clínicos son reproducibles en la práctica clínica diaria. Esto se debe a que los pacientes enrolados son altamente seleccionados, prácticamente no incluyen ancianos y la calidad del seguimiento no es reproducible en el ámbito clínico. Este trabajo representa el estudio prospectivo individual de FA más grande hasta la fecha. Es una cohorte de pacientes ambulatorios reales en el mundo real, que además de incluir un número mayor de pacientes ancianos incluye una mayor cantidad de eventos que los reportados en los otros estudios. ${ }^{5}$

La mayoría de los pacientes fueron seguidos por programas de anticoagulación lo cual probablemente haya contribuido al mejor control de los pacientes anticoagulados, similar al grado de control alcanzado en estudios clínicos.

\section{Conclusiones del Comentador}

La warfarina es efectiva en la práctica clínica diaria para prevenir el ACV en pacientes con FA no valvular con un incremento sólo marginal del riesgo absoluto de hemorragia intracraneal. Este estudio avala el uso de la anticoagulación en pacientes elegibles con riesgo moderado y alto de ACV, particularmente cuando puede realizarse un manejo organizado de la anticoagulación. Los programas de manejo de enfermedades permiten achicar la brecha existente entre la evidencia y la práctica clínica diaria.

Dra. Dorotea Fantl, Dra. Erika Petrlik. Becaria de Hematología [ Sección Hematología Servicio Clínica Médica, Hospital Italiano de Buenos Aires ]

Fantl D, Petrlik E. Los grandes ensayos clínicos de anticoagulación en fibrilación auricular pueden trasladarse a la práctica clínica diaria. Evid. actual. práct. ambul 2004; 7: 167. Comentado de: Go S., Hylek E., Chang Y. et al .Anticoagulation Therapy for Stroke Prevention in Atrial Fibrilation. How well do randomized trials translated into clinical practice. JAMA.2003; 290: 2685-2692.

\section{Referencias}

1.Atrial fibrillation investigators.Risk factors for stroke and efficacy of antithrombotic therapy in atrial fibrillation ;analysis of pooled data from 5 randomized controlled trials Arch Intern Med 1994; 154:1449-1457

2.The Atrial Fibrillation Investigators. The efficacy of aspirin in patients with atrial fibrillation: analysis of pooled data from 3 randomized trials. Arch Intern Med 1997; 157:1237-1240 3. Wyse DJ.,Waldo AL, DiMarco JP, Domanski MJ,Rosenberg Y, Schron EB, Kellen JC, Greene HL, Mickel MC, Dalquist JE, Corley SD N Engl J Med Volume 347:1825-1833 4. Van Gelder, IC., Hagens, VE., Bosker, HA, Kingma, JH., Kamp, O., Kingma,T.,. Said, SA., Darmanata, J.. Timmermans AJ,., Tijssen JG,. Crijns, HJ A Comparison of Rate Control and Rhythm Control in Patients with Recurrent Persistent Atrial Fibrillation N Engl J Med Volume 347:1834-1840

5. Van Walraven C; Hart RG; Singer DE; Laupacis A;Connolly S; Petersen P; Koudstaal PJ; Chang Y; Hellemons B Oral Anticoagulants vs Aspirin in Nonvalvular Atrial Fibrillation: An Individual Patient Meta-analysis JAMA. 2002;288:2441-2448. 